九州大学学術情報リポジトリ

Kyushu University Institutional Repository

\title{
Pdcd4 expression in intraductal papillary mucinous neoplasm of the pancreas : Its association with tumor progression and proliferation
}

Hayashi, Akifumi

Department of Anatomic Pathology, Graduate School of Medical Sciences, Kyushu University

Aishima, Shinichi

Department of Anatomic Pathology, Graduate School of Medical Sciences, Kyushu University

Miyasaka, Yoshihiro

Department of Anatomic Pathology, Graduate School of Medical Sciences, Kyushu University

Nakata, Kohe i

Department of Anatomic Pathology, Graduate School of Medical Sciences, Kyushu University 他

http://hdl. hand le. net/2324/26068

出版情報：Human Pathology. 41 (11)，pp.1507-1515，2010-11. Elsevier バージョン：

権利関係: (C) 2010 Published by Elsevier Inc. 


\title{
Title page
}

Pdcd4 expression in intraductal papillary mucinous neoplasm of the pancreas: its association with tumor progression and proliferation

\author{
Akifumi Hayashi ${ }^{1}$, Shinichi Aishima ${ }^{1}$, Yoshihiro Miyasaka ${ }^{1}$, Kohei Nakata ${ }^{1}$, Katsuya \\ Morimatsu $^{1}$, Yasunori Oda ${ }^{1}$, Eishi Nagai ${ }^{2}$, Yoshinao Oda ${ }^{1}$, Masao Tanaka ${ }^{2}$ and \\ Masazumi Tsuneyoshi ${ }^{1}$
}

${ }^{1}$ Department of Anatomic Pathology, Graduate School of Medical Sciences, Kyushu University, Fukuoka, Japan.

${ }^{2}$ Department of Surgery and Oncology, Graduate School of Medical Sciences, Kyushu University, Fukuoka, Japan.

Keywords: IPMN; Pdcd4; progression; proliferation.

Abbreviations used in this report: Pdcd4, programmed cell death 4; IPMN, intraductal papillary mucinous neoplasm; IPMA, intraductal papillary mucinous 
adenoma; IPMB, borderline intraductal papillary mucinous neoplasm; IPMC,

intraductal papillary mucinous carcinoma

Correspondence should be addressed to: Shinichi Aishima, MD, PhD, Department of Anatomic Pathology, Graduate School of Medical Sciences, Kyushu University 3-1-1

Maidashi, Fukuoka, 812-8582, Japan. Tel +81-92-642-6061, Fax +81-92-642-5968,

E-mail saish@surgpath.med.kyushu-u.ac.jp 
Abstract: Intraductal papillary mucinous neoplasm is characterized by cystically dilated main and/or branch pancreatic duct with mucus. According to the degree of atypia, intraductal papillary mucinous neoplasm is classified into three groups: adenoma, borderline and carcinoma. Furthermore, intraductal papillary mucinous neoplasm is considered to progress through an adenoma-carcinoma sequence like colorectal carcinoma. Programmed cell death 4 is a recently identified tumor suppressor which was found to inhibit translation. Programmed cell death 4 has been reported to inhibit tumorigenesis, tumor progression, proliferation, invasion and metastasis in several human malignancies. We examined 108 cases of intraductal papillary mucinous neoplasm by immunohistochemistry and revealed that programmed cell death 4 expression was recognized in both the nucleus and cytoplasm in intraductal papillary mucinous neoplasm. The positive rate of programmed cell death 4 was $79 \%, 43 \%$ and $10 \%$ in adenoma, borderline and carcinoma, respectively. The positive rate of programmed cell death 4 decreased from adenoma to carcinoma $(\mathrm{P}<0.0001$, both adenoma vs borderline and borderline vs carcinoma), indicating that programmed cell death 4 might inhibit tumor progression in intraductal papillary mucinous neoplasm. Programmed cell death 4 expression had a strong relationship with p21 expression $(\mathrm{P}<0.0001)$ and an inverse correlation with Ki-67 LI $(\mathrm{r}=-0.6255, \mathrm{P}<0.0001)$. Thus, 
Pdcd4 expression in IPMN

A Hayashi et al

programmed cell death 4 might inhibit the proliferation of intraductal papillary

mucinous neoplasm, and its inhibition might partly result from cell cycle arrest caused

by the up-regulation of $\mathrm{p} 21$. In conclusion, programmed cell death 4 may inhibit tumor

progression in intraductal papillary mucinous neoplasm and the loss of programmed cell

death 4 expression is representative of the malignant potential of intraductal papillary

mucinous neoplasm including the proliferative activity. Therefore, programmed cell

death 4 can be an important biomarker for intraductal papillary mucinous neoplasm. 


\section{Introduction}

Intraductal papillary mucinous neoplasm (IPMN) is a cystic tumor in communication with the pancreatic ductal system. The number of IPMN reported is increasing with the increased awareness of this entity and the progress in diagnostic procedures (1).

Macroscopically, this tumor is characterized by cystically dilated main and/or branch pancreatic ducts which are often filled with mucus $(2,3)$. Microscopically, cystically dilated ducts are lined by mucin-producing epithelium with various degrees of atypia, showing a papillary proliferation. According to the degree of atypia, such as architectural and cellular atypia, the WHO has classified IPMN into three groups: intraductal papillary mucinous adenoma (IPMA), borderline intraductal papillary mucinous neoplasm (IPMB) and intraductal papillary mucinous carcinoma (IPMC) (4, 5). IPMN is considered to progress through an adenoma-carcinoma sequence like colorectal carcinoma (6), and the transition from IPMA to IPMB and/or IPMB to IPMC is commonly recognized in certain cases of IPMN. Recently, a number of reports focusing on the molecular and biological changes in IPMN have been published. These reports have revealed that activation of oncogenic pathways such as $K$ ras $(7,8)$ and AKT (9), inactivation of tumor suppressors such as p16, p53 and SMAD4 $(7,10,11)$ and inactivation of the DNA damage checkpoint pathway (12) have been recognized in 
IPMC more frequently than in IPMA or IPMB.

Programmed cell death 4 (Pdcd4) is a novel tumor suppressor gene that was originally identified as a gene up-regulated during apoptosis, and is also known as MA-3 in mice, DUG in rats and H731 in humans (13-15). The human Pdcd4 gene is located on chromosome 10q24 (16) and Pdcd4 protein is expressed in various cells including hematopoietic cells, senescent fibroblasts and normal epithelia of the colon and mammary duct $(15,17-19)$. Up to now, the inhibitory function of Pdcd4 has been described in several human malignancies, such as lung (20), colon $(21,22)$, breast (23), and ovary (24). Although most tumor suppressors target transcription, Pdcd4 is found to target translation by interacting with translation initiation factors eIF4A and eIF4G (25). Down-regulation of Pdcd4 leads to the activation of activator protein-1 (AP-1)-dependent transcription, beta-catenin/Tcf-dependent transcription, and mitogen-activated protein kinase kinase kinase kinase 1 (MAP4K1), and to a decrease of the tissue inhibitor of matrix-metalloproteinase (TIMP), resulting in the inhibition of tumorigenesis, tumor progression, proliferation, invasion and metastasis $(21-24,26)$. Furthermore, using a mice skin carcinogenesis model, Schmid et al. (27) revealed that the down-regulation of Pdcd4 resulted from increased proteasomal degradation of Pdcd4. Thus, Pdcd4 plays a very important role in inhibiting malignant transformation, 
progression and malignant behaviors, and we therefore think that evaluating Pdcd 4 expression may be useful in clarifying the malignant potential of IPMN.

In the present study, we investigated Pdcd4 expression and its subcellular localization in IPMN using immunohistochemistry. By comparing with the degree of atypia, we clarified the significance of Pdcd4 expression in tumorigenesis and tumor progression. Recently, p21 was also reported to be a downstream target of Pdcd4, but the relationship between Pdcd4 and p21 was still under discussion (19, 34, 35). Therefore, we also investigated p21 and p53 expression to reveal their relationship with Pdcd4 in IPMN, and evaluated the Ki-67 labeling index (Ki-67 LI) to reveal the function of Pdcd4 in IPMN. 


\section{Materials and Methods}

\section{Patients and tissue samples}

A total of 108 patients who had undergone surgery for IPMN of the pancreas between July 1986 and October 2006 were included in this study. All samples were fixed in 10\% formalin at room temperature for pathological examination. Whole samples were cut into 4-mm-thick stepwise tissue sections, and all the slices were routinely processed, embedded in paraffin, sectioned and microscopically evaluated for histopathological characteristics. Since IPMN revealed heterologous atypia, we evaluated the lesion showing the highest grade of atypia, such as architectural and cellar atypia and classified IPMN into three groups (IPMA, IPMB and IPMC) according to the WHO classification system (4, 5). Among 108 IPMNs, 39 IPMAs, 30 IPMBs and 39 IPMCs were obtained. Five-year survival rates of IPMA, IPMB and IPMC are 100\%, 100\% and $68 \%$, respectively. 108 normal ductal epithelia beneath the IPMN were also evaluated. Our study protocol conformed to the ethical guidelines of the 1975 Declaration of Helsinki. For strict privacy protection, identifying information for all samples was removed before analysis.

\section{Immunohistochemistry}


Immunohistochemical staining was performed on routinely formalin-fixed and paraffin-embedded tissue sections using the streptavidin-biotin complex method. Briefly, 4- $\mu$ m-thick tissue sections were deparaffinized in xylene and rehydrated through a graded ethanol series. Endogenous peroxidase activity was blocked by incubation in methanol containing $0.3 \% \mathrm{H}_{2} \mathrm{O}_{2}$ for $30 \mathrm{~min}$. The sections were heated in $10 \mathrm{mM}$ citrate buffer ( $\mathrm{pH}$ 6.0) in a microwave oven to retrieve the antigen. After nonspecific binding was blocked by a 10 -min incubation with $10 \%$ rabbit serum, the slides were incubated with primary antibodies at $4{ }^{\circ} \mathrm{C}$ overnight. We used the following primary antibodies: goat polyclonal anti-Pdcd4 (sc-27123; 1:100 dilution; Santa Cruz Biotechnology), mouse monoclonal anti-p21 (EA10; 1:200 dilution; Calbiochem), mouse monoclonal anti-p53 (PAb1801; 1:100 dilution; Oncogene) and mouse monoclonal anti-Ki-67 (MIB-1; 1:50 dilution; R\&D Systems). The antigens labeled by the primary antibody were detected with a Histofine SAB-POkit (Nichirei Pharmaceutical, Tokyo, Japan) and visualized using 3,3'-diaminobenzidine tetrahydrochloride as a chromogen. Nuclei were counterstained with hematoxylin.

\section{Evaluation of immunohistochemical staining}

Because Pdcd4 expression was recognized in both the nucleus and cytoplasm in 
IPMNs, we separately evaluated both nuclear and cytoplasmic staining. We used the nuclear and cytoplasmic scores according to the population of positive nuclear staining cells and the intensity of the cytoplasmic staining, respectively. Referring to previous report in colorectal cancer (31), we set each score as follows (nuclear score: 0\%, score $0 ;<30 \%$, score $1 ; 30-70 \%$, score $2 ;>70 \%$, score 3 and cytoplasmic score: negative, score 0 ; weak, score 1; moderate, score 2; strong, score 3 ). The total score was calculated by adding of the nuclear and cytoplasmic scores. According to the median total score (=3.28), we classified IPMNs into two groups; IPMNs showing a total score of more than 4 were Pdcd4-positive, and the others were negative. For p21 and p53, more than $10 \%$ nuclear staining was considered positive as described previously (11, 28). The Ki-67 labeling index (Ki-67 LI) was calculated by counting Ki-67-positive cells among 1000 tumor cells, according to previous reports (29). In addition, as reported by others $(15,19)$, some hematopoietic cells and fibroblasts in the stroma also had positive staining for Pdcd4, so we used them as the internal positive control.

\section{Statistical Analysis}

All calculations were carried out using StatView 5.0J software (Abacus Concepts, Berkeley, California, USA). Data were analyzed by the Mann-Whitney $U$ test if the 
comparisons involved two groups, because a normal distribution was not obtained. The relationships between Pdcd4 and p21 expression or Pdcd4 and p53 expression were examined using Fisher's exact test. Comparison between the total score of Pdcd4 expression and the Ki-67 LI was performed using Spearman's rank correlation test. A P-value of less than 0.05 was considered statistically significant. 


\section{Results}

\section{Pdcd4 expression in IPMNs}

Pdcd4 expression was recognized in both the nucleus and cytoplasm in IPMNs. Most IPMAs showed strong nuclear and cytoplasmic staining (Fig. 1a). Several IPMCs showed moderate nuclear and cytoplasmic staining (Fig. 1c), but most IPMCs showed weak nuclear and cytoplasmic staining or no staining at all (Figs.1b, 1d). IPMBs revealed intermediate staining (Fig. 1b). Interestingly, most normal ductal epithelia showed weaker staining compared with the IPMAs (Fig. 1a) and the changes of staining were relatively clear in transition areas from IPMB to IPMC (Figs. 1b). The results of the nuclear, cytoplasmic and total scores are shown in Fig. 2. The nuclear and total scores tended to be gradually reduced from IPMA to IPMC $(\mathrm{P}<0.0001$, both IPMA vs IPMB and IPMB vs IPMC). Although the cytoplasmic score also tended to be gradually reduced, the tendency was weaker than that of the nuclear score ( $\mathrm{P}=0.094$, IPMA vs IPMB; P=0.003, IPMB vs IPMC). None of the 108 normal ductal epithelia (0\%), thirty-one of the 38 IPMAs (79\%), thirteen of the 30 IPMBs (43\%) and four of the 39 IPMCs (10\%) were positive for Pdcd4. The positive rate of Pdcd4 also decreased from IPMA to IPMC (Fig. 4a; P=0.0021, IPMA vs IPMB; P=0.0014, IPMB vs IPMC). 


\section{Relationship between Pdcd4 and p21 or Pdcd4 and p53 expression}

Six of the 108 normal ductal epithelia (6\%), thirty-six of the 39 IPMAs (92\%), twenty of the 30 IPMBs (67\%) and seventeen of the 39 IPMCs (44\%) were positive for $\mathrm{p} 21$ (Figs. 3a, 3b, 4b). The expression of p21 tended to gradually decrease from IPMA to IPMC (P=0.007, IPMA vs IPMB; P=0.1845, IPMB vs IPMC), and this tendency was similar to that of Pdcd4 (Figs. 4a, 4b). The expression of p53 was recognized only in IPMCs except for one case of IPMA (Fig. 3c). The positive rate of p53 in normal ductal epithelia, IPMA, IPMB and IPMC were 0\% (0/108), 3\% (1/39), 0\% (0/30) and 23\% (9/39), respectively (Fig. 4c; P=0.003, IPMB vs IPMC ). As shown in Table 1, Pdcd4 expression had a strong relationship with p21 expression $(\mathrm{P}<0.0001)$, but had no significant association with $\mathrm{p} 53$ expression $(\mathrm{P}=0.18)$.

\section{Relationship between Pdcd4 expression and Ki-67 labeling index}

Ki-67 LI showed an increasing tendency from IPMA to IPMC, as expected (Figs. 3d, 5a; $<<0.0001$, both IPMA vs IPMB and IPMB vs IPMC). Although there was no significant association between Pdcd4 expression and Ki-67 LI in IPMAs and IPMBs ( $\mathrm{P}=0.86$ and $\mathrm{P}=0.88$, respectively), Ki-67 LI was significantly higher in the Pdcd4-negative group than in the Pdcd4-positive group when a comparison was 
performed in IPMCs (Fig. 5b; $\mathrm{P}=0.012$ ). Furthermore, we analyzed the correlation between the total score of Pdcd4 expression and Ki-67 LI among all cases, and revealed a significant inverse correlation (Fig. 5c; r=-0.6255, P<0.0001). 


\section{Discussion}

Pdcd4 was a novel tumor suppressor gene that was originally identified as a gene up-regulated during apoptosis (13-15). Pdcd4 differed from many other tumor suppressors in that it targeted translation by interacting with translation initiation factors eIF4A and eIF4G (25). Pdcd4 was also found to inhibit pro-oncogenic events including the activation of activator protein-1 (AP-1)-dependent transcription and beta-catenin/Tcf-dependent transcription (22), resulting in the inhibition of tumorigenesis, tumor progression, invasion and metastasis. In fact, previous studies revealed the inhibitory function of Pdcd4 in various human malignancies, but Pdcd4 expression in the IPMN of the pancreas has not yet been investigated.

In the present study, we evaluated Pdcd4 expression in 108 cases of IPMN using immunohistochemistry. In IPMA, Pdcd4 expression was frequently detected in both the nucleus and cytoplasm, whereas it was significantly reduced in IPMC. The nuclear score tended to be gradually reduced from IPMA to IPMC. The cytoplasmic score showed a similar tendency, but it was weaker than that of the nuclear score. Translation was performed mainly in the cytoplasm, so the cytoplasmic expression of Pdcd4 might be the most important. However, there were several studies showing that Pdcd4 expression was observed in both the nucleus and cytoplasm in normal or cancer samples 
of the colon (31) and breast (32). Furthermore, Palamarchuk et al. (33) revealed that inhibition of AP-1-dependent transcription by Pdcd4 might occur in both the nucleus and cytoplasm. We thus calculated the total score, and based on the median total score, we evaluated the positive rate of $\mathrm{Pdcd} 4$ expression to compare with the other factors. Both the total score and positive rate were significantly reduced from IPMA to IPMC, and most normal ductal epithelia showed weaker staining compared with IPMA. These results were indicative that Pdcd4 was up-regulated in the early stage and might have a greater influence on tumor progression than tumorigenesis in IPMN. The reason why Pdcd4 was up-regulated in the early stage of IPMN was unknown. A possible explanation for this observation was that Pdcd4 might be involved in DNA damage response as well as p53 and p21. Bitomsky et al. (34) revealed that the stable Pdcd4 knockdown cells showed higher survival after ultra-violet (UV) irradiation and were more resistant to UV-induced apoptosis. Therefore, they suggested that low Pdcd4 expression after DNA damage favors the survival of cells. Miyasaka et al. (12) reported that DNA damage check point pathway was activated in the early stage of IPMN. Considering with these result, Pdcd4 might be up-regulated in the response to DNA damage and inhibit the tumor cell survival and proliferation in the early stage of IPMN. In addition, it was interesting that most normal ductal epithelia showed weaker staining 
compared with IPMA. As Schmid et al. (27) showed in HEK293 cells, proteasomal degradation of Pdcd4 might also occur in normal ductal epithelia where Pdcd4 was not yet up-regulated. Moreover, the decreasing tendency from IPMA to IPMC was weaker in the cytoplasmic score of Pdcd4 than in the nuclear score, and there were no cases in which Pdcd4 expression was detected only in the nucleus but not in the cytoplasm, except for five cases of normal ductal epithelia. These results might reflect that Pdcd4 was shuttled from the nucleus to the cytoplasm $(30,31)$ and targeted for proteasomal degradation (27) in accordance with tumor development in IPMN.

p21 was a member of cyclin-dependent kinase inhibitor, whose up-regulation caused cell cycle arrest in both the G1 and G2 phase (19). In the response of DNA damage, up-regulation of p21 occurred to stall G1 or G2 phase until the damage was repaired, and its expression was regulated through both p53-dependent and p53-independent pathways (36). Some studies have identified p21 as a potential target for Pdcd4, but the association between p21 and Pdcd4 has been still controversial. Goke et al. (19) revealed that Pdcd4 induced p21 in Bon-1 cells, whereas Bitomsky et al. (34) showed that knockdown of Pdcd4 resulted in up-regulation of p21 in both HeLa cells and HCT116 cells. And Lankat-Buttgereit et al. (35) showed that the action of Pdcd4 might be cell-type specific. In IPMN, p21 expression exhibited a similar tendency to Pdcd4 
expression. Although there was no significant association between p53 and Pdcd4, p21

had a strong correlation with Pdcd4. These findings suggested that p21 was up-regulated by Pdcd4 independent of p53. p21 was also regulated through p53-independent pathways involving a Myc transcription factor, which was found to inhibit p21 (37). And Pdcd4 was found to inhibit beta-catenin/Tcf-dependent transcription resulting in down-regulation of Myc (38). Thus, up-regulation of p21 might result from inhibition of beta-catenin/Tcf-dependent transcription by Pdcd4. Furthermore, we used Ki-67 LI to assess the relationship between Pdcd4 expression and the proliferative activity of IPMN. Ki-67 LI showed an increasing tendency from IPMA to IPMC, as expected. In IPMA and IPMB, there was no significant association between Pdcd4 expression and Ki-67 LI, but Ki-67 LI was significantly higher in Pdcd4-negative IPMC than in Pdcd4-positive IPMC. Because IPMA or IPMB revealed much lower Ki-67 LI and much smaller differences in Ki-67 LI in each case than IPMC, we might not be able to show the significant association in IPMA or IPMB. Moreover, we also revealed a significant inverse correlation between the total score of Pdcd4 expression and Ki-67 LI. Thus, Pdcd4 might inhibit the proliferation of IPMN, and its inhibition might partly result from cell cycle arrest caused by the up-regulation of p21.

In summary, we exhibited that Pdcd4 expression was reduced with the progression of 
IPMN and had an inverse correlation with the proliferative activity of IPMN. Therefore, Pdcd4 may inhibit tumor progression in IPMN and the loss of Pdcd4 expression is representative of the malignant potential of IPMN including the proliferative activity. Furthermore, Pdcd4 can be an important biomarker for IPMN. 


\section{References}

1. Tanaka M, Kobayashi K, Mizumoto K, et al. Clinical aspects of intraductal papillary mucinous neoplasm of the pancreas. J Gastroenterol 2005;40:669-75.

2. Furukawa T, Takahashi T, Kobari M, et al. The mucus-hypersecreting tumor of the pancreas. Development and extension visualized by three-dimensional computerized mapping. Cancer 1992;70:1505-13.

3. Furukawa T. Molecular genetics of intraductal papillary-mucinous neoplasms of the pancreas. J Hepatobiliary Pancreat Surg 2007;14:233-7.

\section{Longnecker DS, Adler G, Hruban RH, et al. Intraductal papillary-mucinous} neoplasms of the pancreas. In: Hamilton SR, Aaltonen LA, editors. Pathology and genetics of tumors of the digestive system. Lyon: IARC Press; 2000. p. 237-40.

5. Hruban RH, Takaori K, Klimstra DS, et al. An illustrated consensus on the classification of pancreatic intraepithelial neoplasia and intraductal papillary mucinous neoplasms. Am J Surg Pathol 2004;28:977-87.

6. Nagai E, Ueki T, Chijiiwa K, et al. Intraductal papillary mucinous neoplasms of the pancreas associated with so-called "mucinous ductal ectasia". Histochemical and immunohistochemical analysis of 29 cases. Am J Surg Pathol 1995;19:576-89.

7. Satoh K, Shimosegawa T, Moriizumi S, et al. K-ras mutation and p53 protein 
accumulation in intraductal mucin-hypersecreting neoplasms of the pancreas. Pancreas 1996;12:362-8.

8. Kitago M, Ueda M, Aiura K, et al. Comparison of K-ras point mutation distributions in intraductal papillary-mucinous tumors and ductal adenocarcinoma of the pancreas. Int J Cancer 2004;110:177-82.

9. Semba S, Moriya T, Kimura W, et al. Phosphorylated Akt/PKB controls cell growth and apoptosis in intraductal papillary-mucinous tumor and invasive ductal adenocarcinoma of the pancreas. Pancreas 2003;26:250-7.

10. Sessa F, Solcia E, Capella C, et al. Intraductal papillary-mucinous tumours represent a distinct group of pancreatic neoplasms: an investigation of tumour cell differentiation and K-ras, p53 and c-erbB-2 abnormalities in 26 patients. Virchows Arch 1994;425:357-67.

11. Biankin AV, Biankin SA, Kench JG, et al. Aberrant p16(INK4A) and DPC4/Smad4 expression in intraductal papillary mucinous tumours of the pancreas is associated with invasive ductal adenocarcinoma. Gut 2002;50:861-8.

12. Miyasaka Y, Nagai E, Yamaguchi H, et al. The role of the DNA damage checkpoint pathway in intraductal papillary mucinous neoplasms of the pancreas. Clin Cancer Res 2007;13:4371-7. 
13. Goke A, Goke R, Knolle A, et al. DUG is a novel homologue of translation initiation factor 4G that binds eIF4A. Biochem Biophys Res Commun 2002;297:78-82.

14. Shibahara K, Asano M, Ishida Y, et al. Isolation of a novel mouse gene MA-3 that is induced upon programmed cell death. Gene 1995;166:297-301.

15. Yoshinaga H, Matsuhashi S, Fujiyama C, et al. Novel human PDCD4 (H731) gene expressed in proliferative cells is expressed in the small duct epithelial cells of the breast as revealed by an anti-H731 antibody. Pathol Int 1999;49:1067-77.

16. Soejima H, Miyoshi O, Yoshinaga H, et al. Assignment of the programmed cell death 4 gene (PDCD4) to human chromosome band 10q24 by in situ hybridization. Cytogenet Cell Genet 1999;87:113-4.

17. Azzoni L, Zatsepina O, Abebe B, et al. Differential transcriptional regulation of CD161 and a novel gene, 197/15a, by IL-2, IL-15, and IL-12 in NK and T cells. $J$ Immunol 1998;161:3493-500.

18. Kang MJ, Ahn HS, Lee JY, et al. Up-regulation of PDCD4 in senescent human diploid fibroblasts. Biochem Biophys Res Commun 2002;293:617-21.

19. Goke R, Barth P, Schmidt A, et al. Programmed cell death protein 4 suppresses CDK1/cdc2 via induction of p21(Waf1/Cip1). Am J Physiol Cell Physiol 2004;287:C1541-6. 
20. Chen Y, Knosel T, Kristiansen G, et al. Loss of PDCD4 expression in human lung cancer correlates with tumour progression and prognosis. J Pathol 2003;200:640-6.

21. Yang HS, Matthews CP, Clair T, et al. Tumorigenesis suppressor Pdcd4 down-regulates mitogen-activated protein kinase kinase kinase kinase 1 expression to suppress colon carcinoma cell invasion. Mol Cell Biol 2006;26:1297-306.

22. Wang Q, Sun Z, Yang HS. Downregulation of tumor suppressor Pdcd4 promotes invasion and activates both beta-catenin/Tcf and AP-1-dependent transcription in colon carcinoma cells. Oncogene 2008;27:1527-35.

23. Nieves-Alicea R, Colburn NH, Simeone AM, et al. Programmed cell death 4 inhibits breast cancer cell invasion by increasing tissue inhibitor of metalloproteinases- 2 expression. Breast Cancer Res Treat 2009;114:203-9.

24. Wei ZT, Zhang X, Wang XY, et al. PDCD4 inhibits the malignant phenotype of ovarian cancer cells. Cancer Sci 2009;100:1408-13.

25. Yang HS, Jansen AP, Komar AA, et al. The transformation suppressor Pdcd4 is a novel eukaryotic translation initiation factor 4A binding protein that inhibits translation. Mol Cell Biol 2003;23:26-37.

26. Yang HS, Knies JL, Stark C, et al. Pdcd4 suppresses tumor phenotype in JB6 
cells by inhibiting AP-1 transactivation. Oncogene 2003;22:3712-20.

27. Schmid T, Jansen AP, Baker AR, et al. Translation inhibitor Pdcd4 is targeted for degradation during tumor promotion. Cancer Res 2008;68:1254-60.

28. Biankin AV, Kench JG, Biankin SA, et al. Pancreatic intraepithelial neoplasia in association with intraductal papillary mucinous neoplasms of the pancreas:

implications for disease progression and recurrence. Am J Surg Pathol

2004;28:1184-92.

29. Terada T, Ohta T, Kitamura Y, et al. Cell proliferative activity in intraductal papillary-mucinous neoplasms and invasive ductal adenocarcinomas of the pancreas: an immunohistochemical study. Arch Pathol Lab Med 1998;122:42-6.

30. Bohm M, Sawicka K, Siebrasse JP, et al. The transformation suppressor protein Pdcd4 shuttles between nucleus and cytoplasm and binds RNA. Oncogene 2003;22:4905-10.

31. Mudduluru G, Medved F, Grobholz R, et al. Loss of programmed cell death 4 expression marks adenoma-carcinoma transition, correlates inversely with phosphorylated protein kinase B, and is an independent prognostic factor in resected colorectal cancer. Cancer 2007;110:1697-707.

32. Wen YH, Shi X, Chiriboga L, et al. Alterations in the expression of PDCD4 in 
ductal carcinoma of the breast. Oncol Rep 2007;18:1387-93.

33. Palamarchuk A, Efanov A, Maximov V, et al. Akt phosphorylates and regulates Pdcd4 tumor suppressor protein. Cancer Res 2005;65:11282-6.

34. Bitomsky N, Wethkamp N, Marikkannu R, et al. siRNA-mediated knockdown of Pdcd4 expression causes upregulation of p21(Waf1/Cip1) expression. Oncogene 2008;27:4820-9.

35. Lankat-Buttgereit B, Lenschen B, Schmidt H, et al. The action of Pdcd4 may be cell type specific: evidence that reduction of dUTPase levels might contribute to its tumor suppressor activity in Bon-1 cells. Apoptosis 2008;13:157-64.

36. Massague J. G1 cell-cycle control and cancer. Nature 2004;432:298-306.

37. Seoane J, Le H-V, Massague J. Myc suppression of p21Cip1 Cdk inhibitor influences the outcome of the p53 response to DNA damage. Nature 2002;419:729-34.

38. Wang Q, Sun Z-X, Allgayer H, et al. Downregulation of E-cadherin is essential event in activating beta-catenin/Tcf -dependent transcription and expression of its target genes in Pdcd4 knockdown cells. Oncogene 2010;29:128-38. 


\section{Figure Legends}

Figure 1. Pdcd4 expression in IPMN. IPMA shows strong Pdcd4 expression in both the nucleus and cytoplasm, whereas adjacent normal ductal epithelia shows weak Pdcd4 expression (a). IPMB shows intermediate staining (b). Several IPMCs are positive for Pdcd4 (c), but most of IPMC are negative for Pdcd4 (b, d). Furthermore, changes in Pdcd4 expression are relatively clear in transition areas (b).

Figure 2. Result of nuclear (a), cytoplasmic (b) and total score (c) of Pdcd4 expression. Nuclear and total scores show a significant decreasing tendency from IPMA to IPMC ( $<<0.0001$, both IPMA vs IPMB and IPMB vs IPMC). However, the decreasing tendency of the cytoplasmic score is weaker than those of nuclear and total scores ( $\mathrm{P}=0.094$, IPMA vs IPMB; P=0.003, IPMB vs IPMC).

Figure 3. Immunohistochemical staining of p21, p53 and Ki-67. Positive p21 expression in IPMB (a). Negative p21 expression (b), positive p53 expression (c) and high Ki-67 LI (d, Ki-67 LI=58.6) in IPMC.

Figure 4. The positive rates of Pdcd4 (a) and p21 (b) show a decreasing tendency from 
IPMA to IPMC, whereas that of p53 (c) reveals an increasing tendency from IPMA to IPMC.

Figure 5. Ki-67 LI shows an increasing tendency from IPMA to IPMC (a; $\mathrm{P}<0.0001$, both IPMA vs IPMB and IPMB vs IPMC). Ki-67 LI is significantly higher in the Pdcd4-negative group than in the Pdcd4-positive group when a comparison is performed in IPMC ( $b ; \mathrm{P}=0.012$ ). The total score of the Pdcd4 expression and Ki-67 LI reveal a significant inverse correlation among all cases ( $\mathrm{c} ; \mathrm{r}=-0.6255, \mathrm{P}<0.0001)$. 